\title{
STUDY OF LED LAMPS TECHNOLOGIES IMPACT ON THE UTILITY
}

1. Introduction

With the growing interest in the issue of reducing power consumption in electricity applications, energy-saving devises and the problem of their operation gains more attention. The increase in the utilization of energy-saving lamps, especially LED lamps, leads to the wide interest in their influence on the power system. The impact of discussed devices on the voltage and current in the grid must be thoroughly studied before launching devices into the market. The studies undertaken on the LED lamps present in a wide range the issue of harmonic emission from lamps and their influence on power quality. Due to many manufacturing technologies, different LED devices can have various effects on the utility. It is significant to carry out researches concerning the adverse effects of energy-saving lamps in order to utilize devices that meet certain requirements which are set by the international commissions.

The overall purpose of conducted laboratory tests is the wide analysis of different LED tubes influence on power quality with the special emphasis put on the harmonics emission. LED tubes produced by different manufacturers will be studied in the terms of their effect on the utility.

To further understand and judge the influence of LED tubes on the power grid were conducted simulations in Matlab Simulink environment. The simulations concern the issue of harmonics emission from the basic non-linear load which is a diode rectifier with a LED tube. Simulations were done in order to observe the influence of the load on power quality. Subsequently simulations with larger quantities of non-linear devices have been done to understand the operation of many devices connected to the utility and their effect on voltage and current in the grid. In Simulink has been tested and compared different passive filters. It was preceded with the wide analysis of the operation of filters which subsequently allowed to design several passive filters and observe the effects of their utilization in the simulation. In this second part was also discussed the issue of immunity of electrical devices with the emphasis put on the immunity for voltage dips, swells and fluctuations.

In the next step, in the laboratory was built a station to measure the impact of LED tubes on the utility. Devices where tested mainly in the terms of harmonics emission, with the use of various testing equipment. Harmonics emission with different conditions of power suppling was tested in order to observe LED tubes behaviour with various levels of supplied voltage. T

he measuring station was designed with the consideration of parameters of the equipment being under tests and magnitudes which were to be measured. The influence on power quality of different LED tubes have been compared, as well as effects of different LED tubes combinations. Measurements were done with different equipment varying in the ways of measuring and in the accuracy. The station for observing the effects of voltage fluctuations has been designed and built, in order to get the knowledge about LED tubes response for the simulated voltage distortions.

The studies carried out in the laboratory allowed to judge LED tubes influence on power grid and draw conclusions connected with their operation.

Done measurements and calculations allowed to use the gained theoretical knowledge in the practice.

Obtained results led to the complete understanding of the importance of conducted tests and the understanding of obligation of putting emphasis on the issue of power quality being influenced by LED tubes, which are commonly used in the industry. 
2. The principle of LED operation and the issues connected with harmonic disturbances

\subsection{Basics of LEDs operation}

A light-emitting diode is a semiconductor light source that

converts electrical energy into optical energy. The source of lighting is a chip of semiconducting material doped with impurities. Doping a semiconductor leads to the creation of two separate types of semiconductors in the same crystal. The boundary between the two types is a $p-n$ junction. LEDs operation is connected with the phenomenon of electroluminescence which consists of recombination of electrons with holes within the device, accompanied by releasing energy in the form of photons [1].

\subsection{Power quality issue}

Power quality is a worldwide issue as it is connected with continuous supply of electric energy. Electrified and automated modern world is dependent on the uninterrupted supply of power hence the topic of power quality is essential to be studied in a profound way.

The widespread use of electronic equipment, power electronic devices, energy efficient lightning, telecommunications equipment and computerized equipment led to the change of electric loads nature, therefore power quality topics are nowadays causing concerns.

Modern devices are not only susceptible to power quality issues but also they have an influence on current and voltage in the grid. Generally power quality can be understood as the set of parameters defining the properties of electric energy supplied to users or the interaction of electrical power with electrical equipment. It is connected mostly with the parameters of supplied voltage. Nowadays bad power quality is a significant concern of both power suppliers and customers.
There are many terms describing the parameters of power supply that denote its quality, some of which are: voltage sags, voltage interruptions, voltage swells, transients, voltage unbalances, flickers, total harmonic distortion, harmonics, interharmonics, supraharmonics.

The study undertaken on LED tubes showed that the main concern connected with this devices are harmonic disturbances.

\subsection{Generation of harmonics and their effects on the power grid and devices}

The basic reason of harmonics generation is that current flowing through the load is not linearly related to the applied voltage. Harmonics are caused mainly by nonlinear loads, which are used in everyday life. These loads cause current to vary disproportionally with voltage during each period. Nonlinear characteristics of commonly used devices and loads give rise to the harmonic distortion.

Modern electronic equipment draw from the grid nonsinusoidal, distorted current which includes harmonics, which are injected back into power distribution system.

When a non-linear load draws current, that current passes through all of the impedance that is between the load and the system source.

Distorted current afterwards causes distortions in voltage waveform. Harmonics are considered to be the main source of power quality deterioration. They have a number of undesirable effects on the power distribution system and on devices connected to power lines. They contribute to the increase of losses in the distribution grid, affect the operation of electronic equipment, they can also cause failure operation of electronic devices and be the reason of acoustic noise from motors and other machines, as well as with reducing the motors efficiency. 


\subsection{Harmonic standards for LED tubes}

In the terms of power quality it is necessary to define the levels of harmonic distortions. Reduction of harmonics in distribution systems to required levels is mandatory. There exists many standards defining limits of harmonics values. On the international conferences harmonics are being frequently discussed, wherefore many restrictions and standards for harmonic distortions have been created. Devices should meet standards in the terms of emission, they cannot influence power grid to higher than standardized extent.

According to the Standard IEC EN 61000-3-2 the limits of harmonic in current for lighting equipment with $\mathrm{P} \leq 25 \mathrm{~W}$ are presented in the table 1.

Another criteria for lamps having an active input power less than or equal to $25 \mathrm{~W}$ is that third harmonic current cannot exceed $86 \%$ value of fundamental and the fifth harmonic current cannot exceed $61 \%$.

Table 1. Acceptable levels of harmonics for lighting equipment with rated power $P \leq 25 \mathrm{~W}$ in IEC EN 61000-3-2

\begin{tabular}{|c|c|}
\hline $\begin{array}{c}\text { Harmonic } \\
\text { rank } k\end{array}$ & $\begin{array}{c}\text { The maximum permissible } \\
\text { harmonic current calculated for } \\
\text { Watt [mA/W] }\end{array}$ \\
\hline 2 & 3,4 \\
\hline 3 & 1,9 \\
\hline 5 & 1,0 \\
\hline 7 & 0,5 \\
\hline 9 & 0,35 \\
\hline $11 \leq h \leq 39$ & $3,85 / k$ \\
\hline
\end{tabular}

\subsection{Parameters describing the distortion of voltages and currents}

Measurement of power quality usually involves characterising frequency components (in the Standard IEC EN 61000-4-7 frequencies up to $9 \mathrm{kHz}$ ). Harmonics analysis can indicate about power quality but there are a few parameters connected with power quality which are also usually considered during analysis.

\subsubsection{Total harmonic distortion}

Total harmonic distortion (THD) is a measurement of harmonic distortion in a signal.

THD is the ratio of the sum of the harmonic components of signal summed to the specified order of harmonic, to the root meant square value of fundamental component [3]. THD can refer to the distortion of current and voltage waveform. The formula is

$$
T H D=\sqrt{\sum_{h=2}^{n}\left(\frac{Y_{h}}{Y_{1}}\right)^{2}}
$$

where ' $n$ ' in the Standard IEC EN $61000-4-7$ is $40^{\text {th }}$ order harmonic.

\subsubsection{Power factor}

Considering nonlinear load in the terms of power factor issue it is necessary to include the influence of harmonic distortions. There can be distinguished displacement, distortion and true power factor. For currents without harmonic distortion, displacement power factor is equal to the true power factor, which is the ratio of real power to apparent power. With nonlinear load the THD has a value different from zero. It must be taken into account while calculating the power factor.

$$
p f=\frac{P_{a v g}}{U_{1} * I_{1} * \sqrt{1+\left(\frac{T H D_{u}}{100}\right)^{2}} * \sqrt{1+\left(\frac{T H D_{l}}{100}\right)^{2}}}
$$


There can be used some simplifications when the fundamental active power has a close value to the value of active power including higher harmonics components $P_{1 \text { avg }} P_{\text {avg }}$ and when THDU is less than $10 \%$, what is an usual situation. In that case approximate form of true power factor.

$$
p f=\frac{P_{\text {lavg }}}{U_{1} * I_{1}} * \frac{1}{\sqrt{1+\left(\frac{T H D_{I}}{100}\right)^{2}}}=p f_{\text {disp }} * p f_{\text {dist }}
$$

Where $p f_{\text {dist }}$ is distortion power factor connected with harmonics in signal. It is a measure of how much the harmonic distortions generated by the nonlinear load decrease the average power transferred to the load.

The value of displacement power factor can be maximally equal to unity and distortion power factor is always less than one with THD over $0 \%$, so it can be concluded that in nonsinusoidal situation

$$
p f \leq p f_{\text {dist }}
$$

\subsection{Ways of compensating harmonic distortions caused by nonlinear loads}

The compensation of harmonics is overwhelmingly important matter. Harmonics problem are usually resolved by the use of conventional passive and active filters. Other solution is the combination of these two filters, known as hybrid filter. Passive filters relies mostly on the use of inductor (except valley filled circuit with additional diodes) capacitors and resistors.

The classification of passive filters can be done based on the used topology, for example tuned filers or damped filters, or the combination of both. Passive filters can have shunt or series configuration and in various configuration they are usually used for the elimination of current harmonics ant the compensation of reactive power. Damped filters are usually used for the mitigation of all higher harmonics.
They are connected before the load, in shunt or in series. Active filters use special electronic switching equipment which generates harmonic current that cancel the harmonic currents from nonlinear load. Active filters use amplifiers, resistors and capacitors in a feedback loop in order to create the desired filter character.

Overall, the performance of the harmonic cancellation is achieved by injecting equal but opposite current harmonic components and reactive current into power system at the point of connection [2]

In LED tubes used in the industry are mainly used active filters.

\section{Simulations}

Conducted simulations allowed to discuss the issue of emission from LED tube with basic supply circuit, as well as the issue of voltage distortions and the immunity of LED tubes.

The first simulations in Matlab Simulink environment were done in order to observe the LED tube influence on supply voltage and current. The purpose of simulations was harmonics analysis of LED tubes. It has been done using different quantities of LED tubes, to observe their effect on the power quality.

Current and voltage waveforms were observed and the comparison of total harmonic distortions levels in different situations was done. Additionally have been done simulations with commonly used passive filters, to observe their operation in mitigating harmonics. In simulations the effects of using passive filters can be observed and understood.

In Simulink were also simulated voltage disturbances such as voltage fluctuations, voltage swell and drop, undervoltages and overvoltages, to examine LED tube immunity for distortions. 


\subsection{Results of simulations of emission from LED tubes}

Analysed circuit, without power factor correction, consisted of diode rectifier, smoothing capacitor and the LED load. For analysis have been used rectangular window, width $200 \mathrm{~ms}$. Ten intervals lasting $200 \mathrm{~ms}$ each were used to determine THD level.

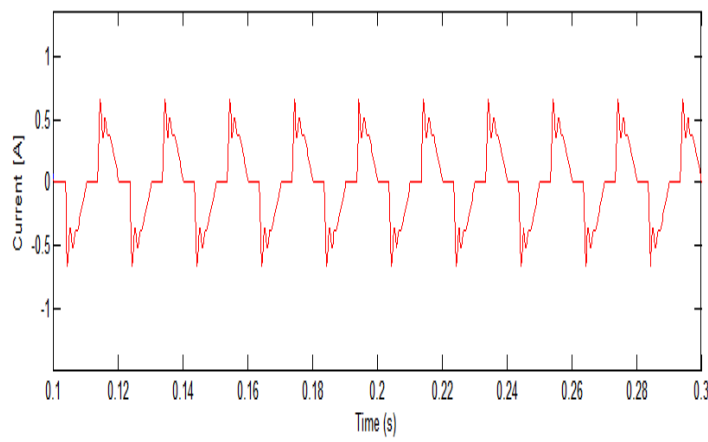

Figure 1. Current waveforms of individual LED tube

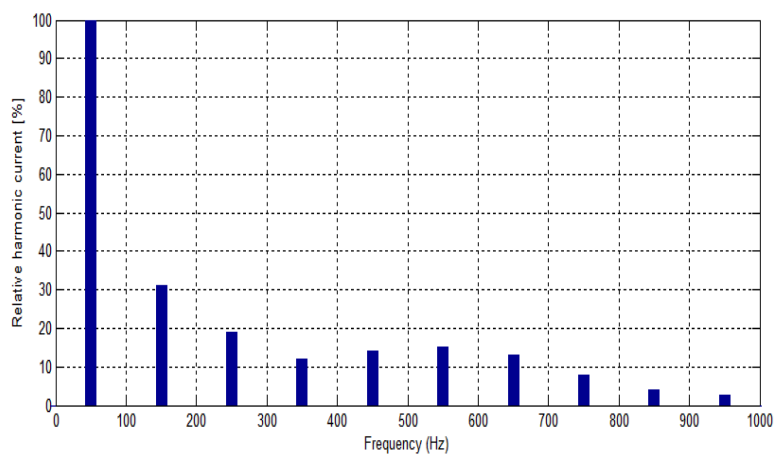

Figure 2. Relative amplitudes of harmonic currents for single LED tube
Current drawn from the grid is highly distorted, thus effected by higher harmonics what is a result of the non-linear characteristic of the load (diode bridge with smoothing capacitor and LED tube).

From the figure 1 it can be noted that the current waveform is not sinusoidal and it means that LED tube inject harmonics into the power system.

Relative amplitudes of harmonic currents of single LED tube are presented in the figure 2 .

In the table 2 are presented results of simulation with different quantities of LED tubes.

It can be concluded that LED tube is the source of harmonics in the grid current. Consequently, voltage in the grid is being distorted. With the increase in the quantity of the same LED tubes up to fifteen LED tubes was observed significant increase in THDU and THDI levels. From twenty LED tubes the THDI started to decrease.

It can be noticed that the decrease of the load's current distortion was accompanied by the increase in the voltage distortions.

The distorted voltage can affect the operation of other loads, in simulated case-the operation of other LED tubes. Addition of other loads led to the increase of THDU and to some quantity of loads, the increase of the THDI.

Table2. Harmonic contents for LED tubes

\begin{tabular}{|c|c|c|c|c|c|c|c|c|c|c|}
\hline $\begin{array}{c}\text { Quantity of LED } \\
\text { tubes }\end{array}$ & $\begin{array}{c}\text { THDI } \\
\text { with } \\
\text { transient } \\
\text { s[\%] }\end{array}$ & $\begin{array}{c}\text { THDI } \\
\text { stable } \\
\text { state [\%] }\end{array}$ & $\begin{array}{c}\text { THDU } \\
\text { with } \\
\text { transient } \\
\text { s\%] }\end{array}$ & $\begin{array}{c}\text { THDU } \\
\text { stable } \\
\text { state [\%] }\end{array}$ & $\begin{array}{c}3^{\text {rd }} \\
{[\%]}\end{array}$ & $\begin{array}{c}5^{\text {th }} \\
{[\%]}\end{array}$ & $\begin{array}{c}7^{\text {th }} \\
{[\%]}\end{array}$ & $\begin{array}{c}9^{\text {th }} \\
{[\%]}\end{array}$ & $\begin{array}{c}1^{\text {th }} \\
{[\%]}\end{array}$ \\
\hline 1 & 83,8 & 46,38 & 4,91 & 1,72 & 31,06 & 18,87 & 12,05 & 13,99 & 15,18 \\
\hline 5 & 78,34 & 54,12 & 11,31 & 7,17 & 31,97 & 36,96 & 22,91 & 2,57 & 2,37 \\
\hline 10 & 79,15 & 63,36 & 16,59 & 12,51 & 53,46 & 33,72 & 0,87 & 2,19 & 2,13 \\
\hline 15 & 77,08 & 64,66 & 19,79 & 15,54 & 61,81 & 18,70 & 1,81 & 2,16 & 1,09 \\
\hline 20 & 76,68 & 55,89 & 25,90 & 17,98 & 54,76 & 10,34 & 3,87 & 0,85 & 1,31 \\
\hline 30 & 62,17 & 51,46 & 24,64 & 20,86 & 50,81 & 6,92 & 3,89 & 1,20 & 1,05 \\
\hline 50 & 38.67 & 31.99 & 26,47 & 21,56 & 30.50 & 6,45 & 2.33 & 1,12 & 1,07 \\
\hline 100 & 33.45 & 28.61 & 24.11 & 15.18 & 28.14 & 4.91 & 1.40 & 0.82 & 0.50 \\
\hline
\end{tabular}


Current in loads increased what is an undesirable effect. The considered reason for this phenomena is that for higher frequencies the input capacitor has a very small impedance, so consequently the value of current increases.

According to the results of simulations it is obvious that circuits without any harmonic filters should not be used in the lighting industry due to the high influence of non-linear load on the power quality.

\subsection{Results of simulation with various passive filters}

In the simulation environment were designed filters: single tuned, double tuned, high pass and C-type. During calculations of filter parameters were chosen exemplary quality factors.

The comparison between three simulated passive filters is shown in the table 3.

Table 3. Results of the mitigation of $3^{\text {rd }}$ and $5^{\text {th }}$ harmonic and THD improvement for simulated filters

\begin{tabular}{|l|c|c|c|}
\hline Passive filter & THDI [\%] & $3^{\text {rd }}[\%]$ & $5^{\text {th }}[\%]$ \\
\hline Two single tuned & 12,21 & 7,25 & 2,97 \\
\hline Double tuned & 13,25 & 5,48 & 1,69 \\
\hline High-pass & 7,09 & 5,27 & 3,10 \\
\hline C-type & 4,84 & 3,70 & 1,64 \\
\hline
\end{tabular}

The best performance with mitigation harmonic distortions was observed with C-type filter and subsequently with highpass filter.

Only the result on decreasing low order harmonic components was compared, so the overall conclusion about passive filter based on this one condition is hard to be drawn and the choice of the filter with the best performance cannot be made.

Omitting aspects such as cost of elements and power losses simplifies the comparison between simulated filters.
It can be concluded that each simulated filter led to the decrease of THDI and THDU what was the purpose of the simulations. The mitigation effect depends on the chosen parameters of filter elements.

\subsection{Results of tests for immunity}

During simulations of suppling LED tape with distorted voltage with visible fluctuations it was observed that rectified voltage and consequently current fluctuates.

The simulated relative changes in the supply voltage did not exceed $10 \%$. In the voltage supply have been observed low frequency interharmonics and subharmonics which are known to be the reason of fluctuations and changes of the amplitude in the LED tube current and consequently changes in the brightness of lamp. This increase in the magnitude occurring with frequency about $20 \mathrm{~Hz}$, may be visible for human eye. The response of LED tube with basic supply circuits for voltage swells, drops, undervoltages and overvoltages was as it was expected. With the increase in voltage, the current increased what in the reality could lead to the change of brightness of lamp or even damage of LED tube. Effect of decreasing the voltage was the decrease in LED tube current.

\section{Laboratory measurements}

Laboratory measurements and further calculations have been done in order to examine LED lamps influence on the utility.

Measurements where done with different equipment which allowed to compare different ways of measuring the impact which have devices on the grid.

Tests were conducted on three LED tubes from various manufacturers, made in the similar technology: Lamp 1, Lamp 2 and Lamp 3. 
Further measurements were done on different combinations of LED lamps to observe the results of connecting various LED tubes in various quantities on power quality. The station to measure harmonic emission had appropriately chosen elements, which allowed to measure harmonics up to $40^{\text {th }}$ order.

\subsection{Results of measurements with various supply voltage}

The first measurements with RIGOL oscilloscope were done in different supply conditions. LED tubes were supplied by $100 \mathrm{~V}, 150 \mathrm{~V}, 200 \mathrm{~V}$ and $230 \mathrm{~V}$. For three LED tubes obtained results were similar.

With the increase of the supply voltage, the distortions in current decreased. For $200 \mathrm{~V}$ and $230 \mathrm{~V}$ the current harmonic distortion were the smallest and the distortion power factor was closets to unity.

Voltage harmonics obtained the highest magnitudes for input voltage $200 \mathrm{~V}$ and above. For Lamp 1 an Lamp 3 LED tubes the highest magnitudes had the $5^{\text {th }}$ and the $7^{\text {th }}$ voltage harmonics.

For Lamp 1 and Lamp 2 LED tube the active power was constant for all situations, however Lamp 3 LED tube achieved rated power with rated voltage supply. With lower voltage active power was lower and the brightness was visibly less.

Results of measurements with $230 \mathrm{~V}$ has been analysed in relation with standards. In rated conditions the voltage THD was the highest for all LED tubes, however the levels were acceptable, they reached more than $2 \%$.

To compare LED tubes influence on power quality, the results of measurements with $230 \mathrm{~V}$ input voltage have been compared.
Table 4. Comparison between LED tubes

\begin{tabular}{|l|c|c|c|}
\hline & Lamp 2 & Lamp 1 & Lamp 3 \\
\hline TDHI [\%] & 19,27 & 10,46 & 16,52 \\
\hline THDU [\%] & 2,28 & 2,13 & 2,01 \\
\hline Grid THDU [\%] & & 0,6 & \\
\hline pftrue [-] & 0,819 & 0,820 & 0,820 \\
\hline pfdist [-] & 0,982 & 0,995 & 0,987 \\
\hline P [W] & 18,71 & 19,07 & 18,68 \\
\hline Sn [VA] & 9,09 & 7,52 & 8,36 \\
\hline
\end{tabular}

Total harmonic distortions from measured devices are shown in the figure 3.

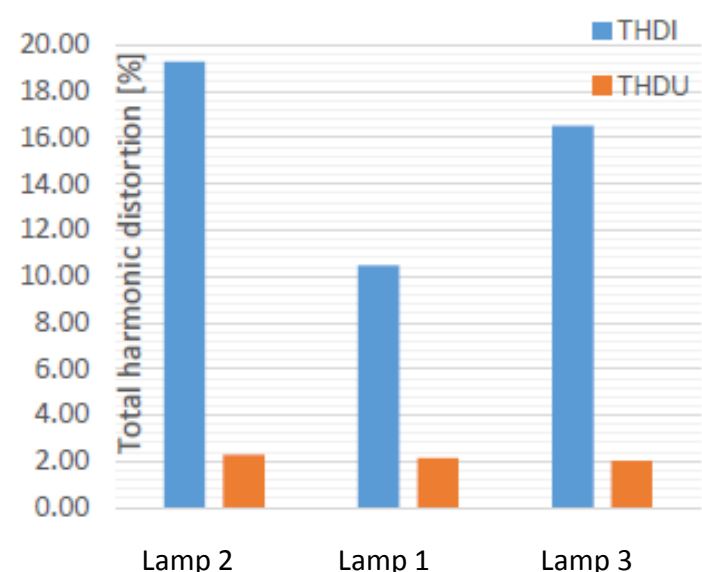

Figure 3. Comparison between three measured LED tubes supplied by $230 \mathrm{~V}$

Each LED tube did not exceed the standardized levels of harmonic currents. Working in rated conditions devices obtained nearly rated power. Power factors are above 0,8 what is a good result and is received due active power filters being internal parts of LED tubes power supplies.

The worst parameters got the Lamp 2 LED tube, however they are still appropriate and have satisfactory levels. This device had also the highest difference between true power factor and displacement power factor, due to the lowest distortion power factor connected with harmonic components. The non-fundamental apparent power was also the highest and it constituted $39,8 \%$ of apparent power. 
The non-fundamental power flow should be as low as possible. Meeting the standards all measured LED tubes can be installed and used without excessive consideration about influence on the power quality.

\subsection{Results of measurements with different LED tubes combinations}

Table 5. Combinations of LED tubes taken under studies

\begin{tabular}{|c|c|}
\hline Combination A & 5 Lamp 2 LED tubes \\
\hline Combination B & 4 Lamp 2 LED tubes + Lamp 1 LED tube \\
\hline Combination C & 4 Lamp 2 LED tubes + Lamp 3 LED tube \\
\hline Combination D & $\begin{array}{l}3 \text { Lamp } 2 \text { LED tubes + Lamp } 1 \text { LED tube+ } \\
\text { Lamp } 3 \text { LED tube }\end{array}$ \\
\hline Combination $\mathbf{E}$ & 3 Lamp 2 LED tubes \\
\hline Combination F & $\begin{array}{l}\text { Lamp } 2 \text { LED tubes+ Lamp } 3 \text { LED tube+ Lamp } \\
1 \text { LED tube }\end{array}$ \\
\hline
\end{tabular}

The level of input voltage was $230 \pm 2 \%$ voltage and the frequency was maintained on the level of $50 \pm 0,5 \% \mathrm{~Hz}$.

In the table 6 are presented results of measurements and calculations for LED tubes combinations with Rigol Oscilloscope.

Table 6. Distortions from different LED tubes combinations

\begin{tabular}{|c|c|c|c|}
\hline Combination & THDU [\%] & THDI [\%] & P [W] \\
\hline A & 1,36 & 20,32 & 101,48 \\
\hline B & 1,70 & 18,69 & 106,51 \\
\hline C & 1,70 & 19,85 & 101,84 \\
\hline D & 1,70 & 18,16 & 101,84 \\
\hline E & 2,04 & 19,34 & 60,09 \\
\hline F & 2,11 & 16,39 & 59,73 \\
\hline
\end{tabular}

Total harmonic distortions from different combinations are compared in the figure 4.

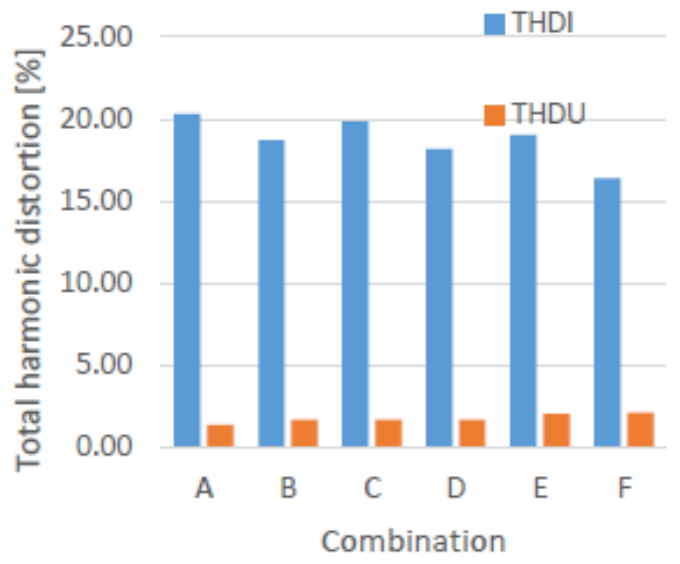

Figure 4. THD for different LED tubes combinations

First four combinations consisted of 5 LED tubes connected in parallel. The magnitude of drawn current has respectively increased. It was observed that with larger quantity of LED tubes the distortions in current increase. Although this LED tubes are made with the same technology and use equal power supply components, the distortions have changed. It is due to the fact that the power supply components have the uncertainties during the operation and they can work with slightly different frequencies, what results in a small increase in harmonic components. THDU for five LED tubes in combination A was smaller than for one LED tube (2,27\%).

It was observed that connecting other LED tubes with Lamp 2 LED tube which influences grid the most, decreases the levels of harmonic distortions. With small quantities of LED tubes it is better to connect different LED tubes to reduce the harmonic distortions. Influence on power grid for each combination is acceptable due to active filters which are parts of every measured LED tube.

Another observed result was that in combination of various LED tubes with changing some LED tubes for another, made with similar technology, the voltage distortion maintains the same.

Measured combinations of 3 LED tubes are characterized with higher voltage distortions than 5 LED tube. 
It is connected with transformers characteristic- with lower load the voltage is more distorted. It can be supposed that with higher quantities of LED tubes, the voltage THD would increase.

\subsection{Comparison of results obtained with different meters}

The use of universal measuring device UMG96S was in order to compare different ways of measuring power quality. The accuracy of oscilloscope used in previous measurements is $\pm 5 \%$ and Janitza measuring device connected with a computer to analyse power quality allowed to measure emission with maximum

Measurements done with Janitza measuring device and further analysis in program Grid Vis gave an accurate results of LED lamps influence on the grid. It was observed that total harmonic distortions measured with UMG96S are higher than measured and calculated with the oscilloscope. Janitza device is appropriate to measure the harmonic components up to $2 \mathrm{kHz}$ with lower uncertainty and allows to study LED lamps influence on power quality in a more accurate way.

The big differences were observed within power factor measurements, especially in displacement power factor. Measurements with UMG96S indicate that LED tubes have almost purely resistive character due to the displacement power factor close to 1 . Results obtained with Rigol Oscilloscope show that displacement power factor is close to 0,8 . It can be due to the simplifications in calculation which may have led to significant differences in true power factor measurement with two devices, however results obtained with Janitza meter are more reliable and should be understood as true values.

The differences between power factor led to noticeable differences in the reactive power measurement. It can be concluded, that UMG96S indicates higher influence of LED tubes on power quality, but on the other side shows better quality of LED tubes due to the higher active power and true power factor.
In the table 7 are presented the results of measurements with LED tubes combinations, done with JANITZA device.

Table 7. Parameters of LED tubes combinations obtained with UMG96S

\begin{tabular}{|c|c|c|c|}
\hline Combination & $\begin{array}{c}\text { THDU } \\
{[\%]}\end{array}$ & $\begin{array}{c}\text { THDI } \\
{[\%]}\end{array}$ & P [W] \\
\hline A & 2,27 & 21,13 & 99,87 \\
\hline B & 2,40 & 18,70 & 98,92 \\
\hline C & 2,42 & 20,35 & 97,90 \\
\hline D & 2,38 & 18,75 & 98,76 \\
\hline E & 2,45 & 21,00 & 59,23 \\
\hline F & 2,55 & 18,10 & 58,92 \\
\hline
\end{tabular}

Figure 5 shows the comparison between results obtained with two used meters.

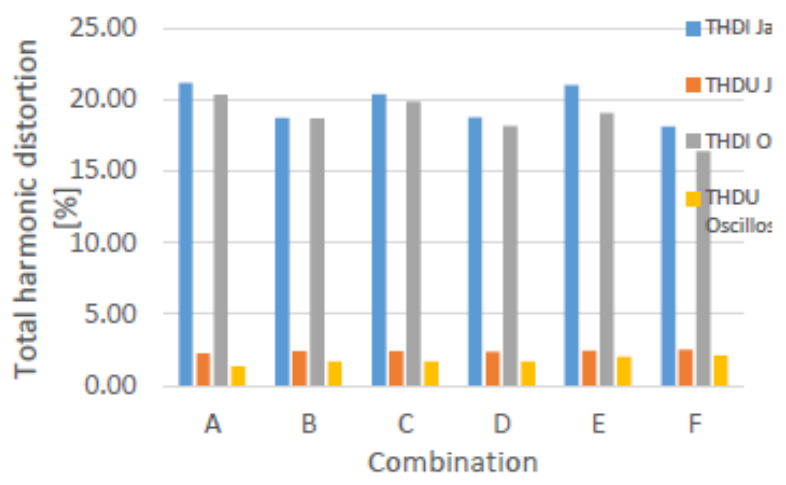

Figure 5. Comparison between levels of THD for various combinations of LED tubes tested with different equipment

Test of various LED tube combinations with UMG96S lead to similar conclusions as the results of the measurements with the oscilloscope.

The levels of THDU and THDI were higher than measured with oscilloscope (the same was observed for the individual lamps), however the same conclusions connected with harmonic emission by different tubes combinations as with previous measurements can be drawn. 


\subsection{LED tubes responses for voltage fluctuations}

The behaviour of LED tubes during voltage oscillations has been observed and the electric parameters have been measured in order to observe the changes in LED tubes operation during voltage disturbances.

The concept of producing voltage fluctuations was based on the relay which connected and disconnected the resistance on the secondary of transformer, with appropriately chosen frequency, to produce voltage drop.

Flicker occurring with following frequencies was tested: 0.5 $\mathrm{Hz}, 1 \mathrm{~Hz}, 2.5 \mathrm{~Hz}, 3.5 \mathrm{~Hz}, 5 \mathrm{~Hz}, 6 \mathrm{~Hz}, 8.8 \mathrm{~Hz}, 10 \mathrm{~Hz}, 12 \mathrm{~Hz}, 15 \mathrm{~Hz}$, $18 \mathrm{~Hz}, 20 \mathrm{~Hz}$. With changing the resistance on the primary site of the transformer, the level of voltage fluctuations was changed.

With lower resistance on the primary site the voltage changes during process of connecting and disconnecting the second resistor were less, the maximum voltage drop was $4 \%$. With higher resistance on the input, the voltage changes were higher, they reached $7 \%$. With less input resistance was observed lower flicker in the LED tube.

LED tube response for voltage fluctuations was visible as a flicker. The results of test can be only discussed due to individual observation of changes in lamp brightness. To measure flicker indicators is demanded special equipment which measures directly short and long term flicker indicators.

The results of tests were similar for three LED tubes. The most visible flicker has been observed for frequency $15 \mathrm{~Hz}$. With increasing the resistance on the primary, the flicker was more visible and it started to be very disturbing with the frequency $3.5 \mathrm{~Hz}$. With lower input resistance, the flicker was lower and it started to be very disturbing with the frequency $5 \mathrm{~Hz}$.
LED tubes are likely to produce visible flicker because of the not immediate reaction of power supply for voltage changes. Switched mode power supply keeps constant current in LED tubes, but it needs very short time to adjust to changes in voltage. During this short period of time, the current in LED tube is slightly changing what lead to the visible changes in the brightness of lamp.

\section{Conclusions}

The purpose of the project was to analyse LED tubes impact on the power grid as well as understand the problem of power quality and ways of its improvement.

The issue of emission from LED lamps was studies and the topic of LED tubes immunity was raised. The issue of harmonics emission is nowadays very significant in the terms of power quality.

LED tubes tested in the laboratory met all required standards. Harmonics emission did not exceed acceptable levels.

LED tubes influence voltage and current in the grid due to their nonlinearity, however this impact is being controlled with the use of mitigation techniques.

Modern LED tubed witch switched mode power supply consist of active filter which is an integral part of the supply. The filter efficiently lowers harmonics emission and distortions caused by LED tubes.

Considerations taken in the simulation part led to the conclusion that LED lamps supplied with circuits without harmonics filters influence the utility to the high extent. Harmonic distortions produced by LED tubes can have negative effect on other devices connected to the grid and for that reason the emission must be maintained below certain levels. 
To obtain satisfactory results of LED tubes influence on the power grid in the simulation, the passive filters were simulated. The use of passive filters led to the significant improvement of power quality.

Laboratory measurements showed that LED tubes influence on the power grid is depended on the technology in which device is produced.

Each of tested lamps had a similar power supply, however differences in the components led to differences in harmonics emission. The power quality was an overall subject of studies with two different meters.

The use of various measuring equipment allowed to compare obtained results and draw more accurate conclusions about emission from devices and about LED tubes parameters. LED tubes produce harmonic distortions.

The high frequency radiation from switched power supply has been also checked, however the emission from devices in frequencies over $2 \mathrm{kHz}$ should be further tested in future measurements.

The behaviour of LED tubes in disturbed conditions has been tested and it was observed that LED tubes are likely to flicker.

Changes in the voltage led to visible changes in the brightness of lamps, despite the use of constant current power supply. Fluctuations in voltage were produced only to observe and compare the LED tubes response, which was the same with all tested tubes.

Larger quantities of LED tubes have been tested and it was observed that their influence on the power grid was quite similar to the influence of individual LED tubes.

Differences in measurement results were depended on the quantity of connected devices and the manufacturers of devices, however none of the tested combinations did exceed standard levels. This is also due to the active filters which efficiently mitigate harmonics.

The study of LED tubes impact on the power grid led to the understanding of power quality issues and the importance of undesirable distortions mitigation.

LED tubes used in the industry should be properly designed and tested in the terms of harmonic emission.

Devices tested in the project influence voltage and current in the grid, however to the admissible extent.

To fully confirm the impact of devices on the utility, the larger amounts of LED tubes should be tested, however this could be a subject of another project, considering the issue of large amounts of devices impact on the power quality.

\section{LITERATURE}

[1] Byungcheul Kim, Eui-Seok Jeon, LED Lighting System Switched-Mode Power Supply, Engineering and Technology (S-CET), 2012 Spring Congress on, pp. 1-3, 2012.

[2] Sandoval Gonzalo, Houdek John, A Review of Harmonic Mitigation Techniques, 2005.

[3] Electromagnetic compatibility (EMC) - Part 4-7: Testing and measurement techniques - General guide on harmonics and interharmonics measurements and instrumentation, for power supply systems and equipment connected thereto (IEC 61000-47:2002/A1:2008) 